

\title{
COMPORTAMIENTO Y CONFORT TÉRMICO EXTERIOR DE CANALES VIALES URBANOS INSERTOS EN DIVERSAS TRAMAS Y GEOMORFOLOGÍAS DE MENDOZA.
}

\author{
THE BEHAVIOR AND OUTDOOR THERMAL COMFORT OF URBAN \\ CANYONS IN DIFFERENT WEFT AND GEOMORPHOLOGIES OF \\ MENDOZA, ARGENTINA.
}

ANA LAURA CASTILLO

Arquitecta

Instituto de Ambiente, Hábitat y Energía, Consejo Nacional de Investigaciones Científicas y Tecnológicas (CONICET)

Mendoza, Argentina

https://orcid.org/0000-0001-8240-455

alcastillo@mendoza-conicet.gob.ar

ERICA NORMA CORREA

Ing. Química -Doctora

Consejo Nacional de Investigaciones Científicas y Tecnológicas (CONICET), Mendoza, Argentina

https://orcid.org/0000-0003-1690-076X

ecorrea@mendoza-conicet.gob.ar
MARÍA BELÉN SOSA

Doctora en Arquitectura

Consejo Nacional de Investigaciones Científicas y Tecnológicas

(CONICET), Mendoza, Argentina

http://orcid.org/0000-0002-0141-442X

msosa@mendoza-conicet.gob.ar

María Alicia Cantón

Arquitecta

Consejo Nacional de Investigaciones Científicas y Tecnológicas (CONICET), Mendoza, Argentina

https://orcid.org/0000-0002-8714-9697

macanton@mendoza-conicet.gob.ar

\section{RESUMEN}

Este trabajo se desarrolla en el Área Metropolitana de Mendoza (AMM), ciudad de clima árido con dos contextos geomorfológicos diferentes. El objetivo consiste en analizar la relación entre el comportamiento y confort térmico exterior de canales viales urbanos (CVU) insertos en llanura y piedemonte. Para ello, se seleccionó y monitoreó seis CVU insertos en tres formas de trama (racional, orgánica y Cul-de-Sac). Los resultados en cuanto al comportamiento térmico indican que el CVU racional es más caluroso en piedemonte que en llanura; el CVU Cul-de-Sac es más caluroso en llanura que en piedemonte; y el CVU orgánico registra la temperatura máxima más elevada pero la temperatura mínima más fresca en llanura. En términos de confort exterior, el CVU racional en llanura alcanza un 30\%, y en el sector de piedemonte representa un $20 \%$; el CVU orgánico en piedemonte alcanza un 30\%, y en el sector de llanura alcanza un 20\%; siendo el CVU Cul-de-Sac en piedemonte el de mejor habitabilidad (70\%); mientras que en llanura alcanza solo un $20 \%$. Esto demuestra que la respuesta térmica y el grado de confort de los CVU insertos en una misma trama urbana tienen diferentes comportamientos de acuerdo a la geomorfología de implantación.

Palabras clave

confort térmico exterior, trama urbana, llanura, piedemonte.

\section{ABSTRACT}

This research was carried out in the Mendoza Metropolitan Area (MMA), a city with an arid climate and two different geomorphological contexts. The objective is to analyze the relationship between the behavior and outdoor thermal comfort of urban canyons (UC) located on flatland and hillsides. To this end, six UC were selected in three urban layouts (grid, organic and cul-de-sac) and monitored. The results regarding thermal behavior indicate that the grid UC is hotter on hillsides than on flatland; the cul-de-sac UC is hotter on flatland than on hillsides; and the organic UC has the highest maximum temperature but the coolest minimum temperature on flatland. In terms of outdoor thermal comfort, the grid UC on flatland reaches $30 \%$, and in the hillside area 20\%; and the organic UC on the hillside reaches $30 \%$, and in the flatland area $20 \%$. The case with the best habitability was the cul-de-sac UC on the hillside, with thermal comfort reaching $70 \%$, while only $20 \%$ in the cul-de-sac UC on flatland. This shows that the outdoor thermal behavior and comfort of the UCs in the same urban layout have different behaviors according to the geomorphology of their location.

Keywords outdoor thermal comfort, urban layout, flatland, hillside. 


\section{INTRODUCCIÓN}

El modelo de desarrollo de las áreas urbanas en Latinoamérica ha seguido, durante las últimas décadas, directrices de planificación dominadas principalmente por la valoración económica inmobiliaria especulativa del suelo (Encinas, Marmolejo y Aguirre, 2016; Castrillón y Cardona, 2014; Libertun de Duren, 2014). Esta situación ha generado que los factores sociales y especialmente los ambientales hayan sido relegados, generando así un desequilibrio en el desarrollo de las áreas urbanas y, en algunos casos, un bajo nivel de eficiencia en el uso y preservación de los recursos naturales propios de cada territorio (Ko y Radke, 2014; Dobbs, Hernández y Reyes, 2018).

El crecimiento de las ciudades, a nivel mundial, ha estado acompañado por fuertes cambios en sus características espaciales, un proceso tendiente a la configuración de un modelo cada vez más disperso del territorio. Angel et al. (2011) detectaron que el crecimiento de la población en el siglo XX fue del $1.6 \%$ anual, mientras que el aumento del uso del suelo fue del $3.6 \%$, produciéndose una disminución de la densidad. Este escenario ha alertado a generar y promover que las existentes y nuevas urbanizaciones sigan patrones de desarrollo sostenibles; constituyendo éste el objetivo número 11, "Ciudades y Comunidades Sostenibles", del Informe de los Objetivos de Desarrollo Sostenible planteado como meta a escala mundial para el año 2030 (UN-Hábitat, 2016; Siclari, 2017).

Las diversas formas de desarrollo y crecimiento de las áreas urbanas modifican las condiciones climáticas naturales propias del sitio de inserción (Mohammed y Chang, 2015; Mawromatis, 2013; Delgado, Campos Chávez y Rentería Juárez, 2012). La transformación de las superficies permeables a impermeables altera localmente los balances de energía haciendo que las ciudades sean más calientes que las áreas rurales perimetrales y dando origen al fenómeno denominado isla de calor urbana (ICU) (Akbari et al., 2016; Levermore et al., 2018; Oke, 1982). Dicha ICU impacta sobre varios factores. Una de las consecuencias más notorias en el balance térmico de las personas es la disminución del grado de habitabilidad térmica de los espacios exteriores (Sun et al, 2017; Tumini, Higueras y Baereswyl Rada, 2016; Tumini, 2013).

En este contexto, varios estudios plantean el desafío de diseñar espacios exteriores confortables que fortalezcan el uso e interacción en las ciudades y que, al mismo tiempo, disminuyan los efectos negativos de la urbanización (Salas Esparza y Herrera Sosa, 2017; Tumini y Pérez, 2015). En efecto, el confort térmico de las personas, en espacios exteriores, es uno de los factores que más influye en la habitabilidad de los espacios públicos de las ciudades (Gómez, Higueras y Ferrer, 2016; Taleghani et al, 2015; Guzmán y Ochoa de la Torre, 2014; Nikolopoulou y Lykoudis, 2006). Los estudios de Givoni (1989), en este sentido, han demostrado cómo la cantidad e intensidad de actividades que un individuo realiza es afectada por el nivel de incomodidad térmica experimentado cuando se expone a las condiciones climáticas en espacios abiertos.

Así, ciertas características urbanas pueden contribuir a mejorar el grado de habitabilidad térmica exterior. La literatura internacional sobre estas temáticas aborda la evaluación del comportamiento y confort térmico de las ciudades, centrándose en el estudio a escala de canal vial urbano (CVU), ya que en entornos construidos los CVU suelen componer más de una cuarta parte de las áreas urbanizadas (Shashua-Bar y Hoffman, 2003; UN-Hábitat, 2013). Existen numerosos estudios a nivel mundial que investigan $y$ discuten cómo ciertas variables que conforman el espacio urbano colaboran a reducir las temperaturas exteriores a escala de CVU (Sanusi et al., 2016; Lin, Matzarakis y Hwand, 2011; Shashua-Bar, Pearlmutter y Erell, 2009; Emmanuel y Fernando, 2007; Golden, 2004; Pearlmutter, Bitan y Berliner, 1999). En relación a ello, el abordaje científico ha avanzado parcialmente a partir de investigaciones que han demostrado, a escala de barrio, que la forma urbana tiene un fuerte impacto en las temperaturas diurnas (Middel et al., 2014; Middel, Chhetri y Quay, 2015). Setaih, Hamza y Townshend (2013) realizan una revisión bibliográfica extensiva de estrategias de mejora térmica que pueden ser implementadas por urbanistas, tales como: materiales reflectantes fríos, superficies de agua, espacios verdes y vegetación, y elementos de sombreado, entre otros. Barakat, Ayad y El-Sayed (2017), en tanto, indican que el uso de vegetación, la selección de materiales apropiados, y la utilización de elementos de agua (fuentes) mejoran el confort térmico en escenarios propuestos con reducciones de hasta $3^{\circ} \mathrm{C}$.

A nivel local, Ruiz et al. (2015; 2017) y Alchapar y Correa (2015) han evaluado distintas estrategias de reducción de las temperaturas ambientales a escala de CVU. A su vez, Sosa, Correa y Cantón (2017a) consideran a la forma de agrupamiento de las edificaciones "trama + perfil urbano", como estrategia de planificación para contribuir al enfriamiento de las temperaturas del aire urbano. Los resultados demuestran que para el Área Metropolitana de Mendoza (AMM), la combinación entre las estrategias de reducción térmica aplicadas al CVU y a la forma de la trama urbana, alcanzan diferencias de $2.5^{\circ} \mathrm{C}$ en la temperatura promedio de los conjuntos entre las alternativas evaluadas. Y aunque las estrategias son similares a las utilizadas a nivel internacional, la magnitud de su impacto y su eficiencia en el AMM varía; lo cual revela que los resultados son dependientes de las particularidades de la ciudad (clima, sitio de implantación, forma, materialidad, vegetación).

El Manual de Sustentabilidad en el Espacio Público, generado por el Ministerio de Vivienda y Urbanismo (MINVU) (2017), señala que el confort térmico en espacios abiertos está influenciado por diversos factores: condiciones microclimáticas, forma urbana, actividades metabólicas de los usuarios y materialidad del espacio exterior (Figura 1). A partir del análisis de la Figura 1 se infiere que ciertas variables que interactúan con la forma urbana pueden 

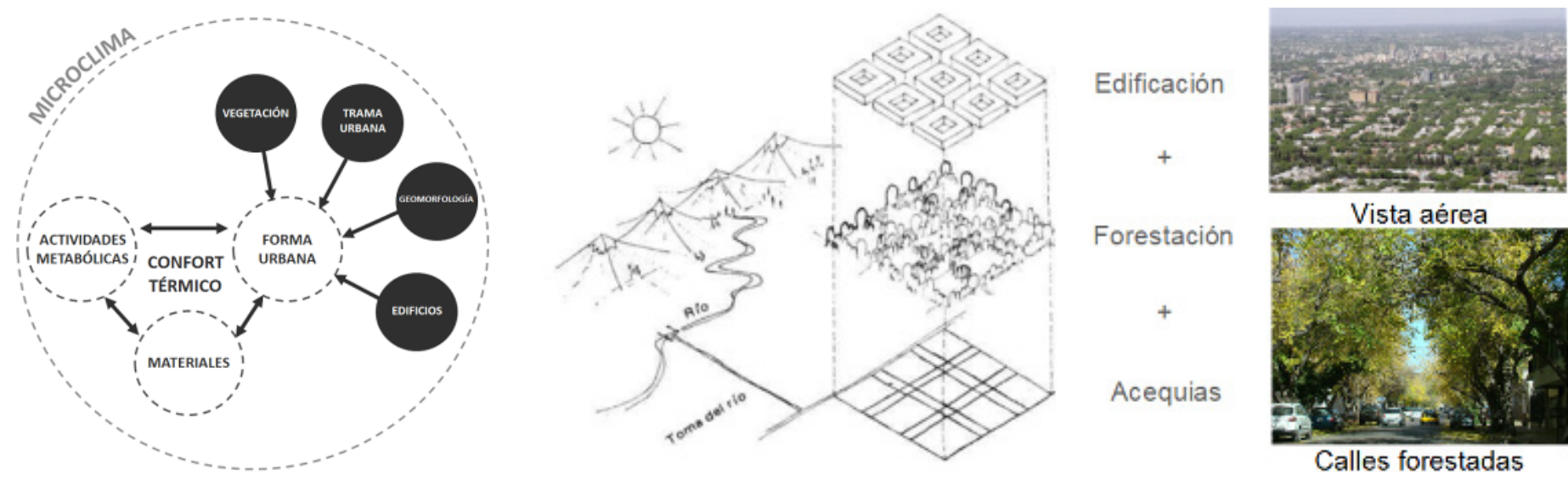

Calles forestadas

Figura 1. Factores que determinan el confort térmico de una persona y variables que interactúan con la forma urbana. Fuente: Adaptado por las autoras a partir de MINVU (2016).

Figura 2. Superposición de estructuras de la "ciudad oasis". Fuente: Elaboración de las autoras a partir de Bórmida (1984).

alterar las condiciones de confort térmico exterior, entre ellas la geomorfología. Se entiende por "geomorfología" a la variedad de formas del relieve que existen en una superficie de territorio, siendo el relieve un factor de caracterización ambiental relevante ya que condiciona fuertemente las posibilidades de uso del territorio (Abraham, 1996).

Diversos estudios destacan la importancia de considerar la geomorfología en los procesos de planificación del territorio, debido a que éstos plantean limitaciones al desarrollo urbano (Villegas et al., 2015; Cavallaro, Nicosia Burgos y Fontaneto, 2010). Considerables problemas ambientales podrían evitarse si las nuevas urbanizaciones se proyectasen y construyesen teniendo en cuenta las condiciones geomorfológicas propias del terreno a intervenir (Albă, Mititelu-ionuş, y Boengiu, 2017). Esto se refleja, por ejemplo, en la modificación de las trayectorias naturales de vientos y brisas locales, así como en la capacidad de absorción y acumulación de calor del terreno natural (Wang y Oayang, 2016; Castaldo, et al., 2017), lo cual afecta también la infiltración y escorrentía de las precipitaciones (Vich et al., 2014).

Para el caso de estudio, AMM, ciudad de clima árido localizada en el centro oeste argentino, la geomorfología en la extensión del territorio presenta dos escenarios fundamentalmente distintos: llanura (altitud entre 300 y 800 m.s.n.m.) y piedemonte (altitud entre 801 y 1400 m.s.n.m.). En ambos entornos geomorfológicos, los esquemas de desarrollo urbano son de tipo "ciudad oasis", que representa un caso particular en el urbanismo de zonas áridas, ya que ahí los CVU son anchos, tienen forestales de alineación y las construcciones son de baja altura (1-2 niveles, baja densidad). Este modelo peculiar de urbanización busca acondicionar ambientalmente un sector de desierto, mediante una macro-estructura espacial, que consta esencialmente de una red de riego y un entramado de árboles, superpuesta a la estructura arquitectónica (Figura 2).
Desde hace más de 10 años que se documenta en el AMM la existencia de la ICU y sus consecuencias asociadas. La ICU registra incrementos de temperatura máxima del orden de $10^{\circ} \mathrm{C}$ (Correa, De Rosa y Lesino, 2008). Los estudios a nivel regional demuestran que este fenómeno provoca durante el verano que sus espacios públicos y CVU se encuentren en incomodidad térmica por calor durante el 46 al $62 \%$ del tiempo, con la consecuente disminución del grado de habitabilidad y uso de los mismos (Correa, Ruiz y Cantón, 2010; Stocco, Cantón y Correa, 2015; Sosa, Correa y Cantón, 2017b). Sin embargo, aún no se evidencia a nivel local, el abordaje del impacto térmico exterior que ejerce la forma de CVU contenidos en diversas tramas urbanas, en relación a su geomorfología de implantación.

En este contexto, el presente trabajo tiene como objetivo monitorear y analizar, durante el periodo estival, el comportamiento térmico exterior y estimar el grado de confort a escala de CVU. A partir de ello, se propone determinar si existe alguna relación en cuanto a la forma de los CVU insertos en los dos escenarios geomorfológicos del AMM, que permita generar recomendaciones de diseño a urbanistas y planificadores.

\section{MARCO DE PLANIFICACIÓN SEGÚN ESCENARIO GEOMORFOLÓGICO}

El crecimiento urbano del AMM está regulado por dos leyes provinciales: la Ley de loteo o fraccionamiento $\mathrm{N}^{\circ} 4341$ y la Ley de disposición del suelo de zona oeste del gran Mendoza No 4886. La Figura 3 contrasta ambas normativas a través de un conjunto de descriptores e indicadores urbanos -ancho de calle, superficie mínima de parcela, frente de la parcela, altura máxima y factor de ocupación del suelo (FOS)-, diferenciados según zonas urbanas y sub-urbanas. Ello, a pesar de que esta condición de diferenciación territorial es poco clara y no existe cartografía específica que delimite ambos sectores dentro del AMM. 


\begin{tabular}{|c|c|c|c|c|}
\hline \multirow{3}{*}{\begin{tabular}{|l} 
Escenario \\
Ley \\
Zonificación
\end{tabular}} & \multicolumn{2}{|c|}{ Llanura } & \multicolumn{2}{|c|}{ Piedemonte } \\
\hline & \multicolumn{2}{|c|}{ Loteo de terrenos $\mathrm{N}^{\circ} 4341$} & \multicolumn{2}{|c|}{ Disposición zona oeste $\mathrm{N}^{\circ} 4886$} \\
\hline & Urbana & Sub-urbana & Urbana & Sub-urbana \\
\hline Ancho calle (m) & $16-20-30$ & $16-20-30$ & 16 & 16 \\
\hline Sup. mínima de parcela $\left(\mathrm{m}^{2}\right)$ & 200 & 200 & 200 & 500 \\
\hline Frente parcela (m) & 12 & 12 & 14 & 14 \\
\hline Altura máxima (m) & Según zona & Según zona & 10 & 10 \\
\hline FOS & $\begin{array}{c}\text { Según zona } \\
\text { (mín } 0.40, \text { máx } 1.00)\end{array}$ & $\begin{array}{c}\text { Según zona } \\
(\text { mín } 0.30, \text { máx } 0.60)\end{array}$ & 0.35 & 0.35 \\
\hline
\end{tabular}

Figura 3. Comparación de las variables urbanas según dos leyes provinciales vigentes. Fuente: Elaboración las autoras.

En la Figura 3, se aprecia que, en ambos escenarios -llanura y piedemonte-, no existe diferenciación en el modo de ocupación de territorio para distintos usos -zonas urbanas y sub-urbanas- ni para los distintos relieves de territorio. Sólo se observa, en el caso del piedemonte, una variación en la superficie mínima de parcela según usos $\left(200 \mathrm{~m}^{2}\right.$ vs. 500 $\mathrm{m}^{2}$ ) y que los anchos de CVU toman el valor mínimo $(16 \mathrm{~m})$. Esto podría ser un promotor importante para fomentar la compacidad de la trama y así disminuir la exposición solar de los CVU, como también para generar mayores superficies de suelos permeables en escenarios como el piedemonte que poseen alta fragilidad ambiental, grandes pendientes, lluvias estivales concentradas, vegetación escasa y degradada, suelos no consolidados y factibles de padecer acelerados procesos de erosión, principalmente hídrica (Abraham, 1990).

Como resultado, el desarrollo urbanístico de piedemonte replica en gran medida el esquema urbano de llanura, sin advertir que en estos sectores las respuestas de planificación y diseño de CVU deben adaptarse a las características inherentes del territorio a intervenir.

De lo descrito se desprende que en el área de estudio la componente ambiental y de adaptación al escenario natural de interacción pareciera no estar considerada dentro de los lineamientos legales de planificación. En consecuencia, esta situación afecta al comportamiento térmico de los desarrollos urbanos, incrementa los consumos de energía e impacta negativamente en las condiciones de confort de los espacios abiertos.

\section{METODOLOGÍA}

La metodología del trabajo se divide en cuatro secciones, a saber: (i) selección y descripción de los casos de estudio en llanura y piedemonte; (ii) monitoreo y adquisición de datos microclimáticos; (iii) cálculo de las condiciones de confort térmico exterior con el modelo COMFA -COMFort formulA(Brown y Gillespie, 1995); y, (iv) correlación estadística, coeficiente de Pearson, entre los indicadores urbanos y el comportamiento térmico y de confort.

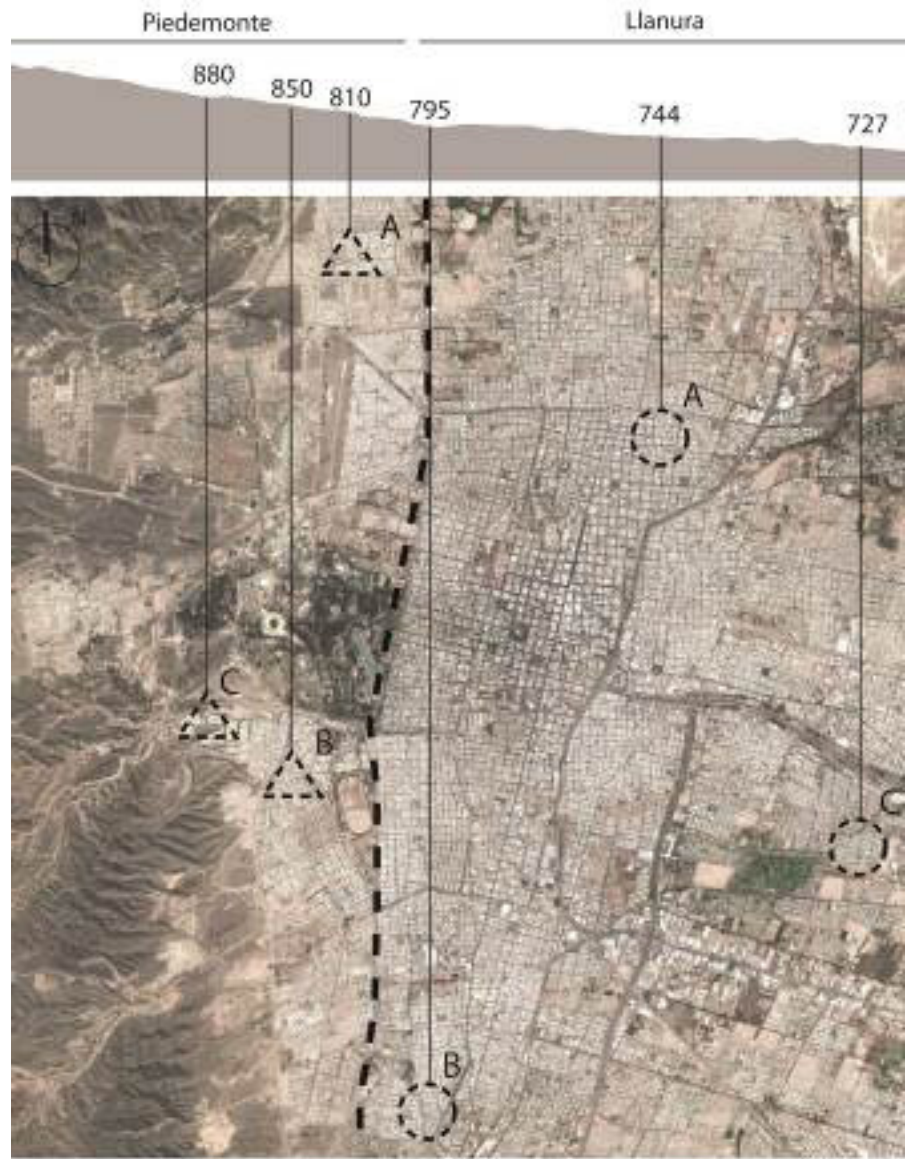

A_Trama Racional B_Trama Orgánica C_Trama Cul de Sac
Figura 4. División sector llanura y piedemonte y ubicación casos de estudio A) trama racional; B) trama orgánica y C) trama Cul-de-Sac. Fuente: Elaboración de las autoras. 


\begin{tabular}{|c|c|c|c|c|c|c|}
\hline Trama & & & Org & & & \\
\hline & Llanura & Piedemonte & Llanura & Picdemonte & Llamura & Piedemonte \\
\hline Escenario & 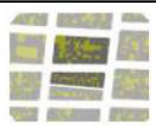 & (5) & 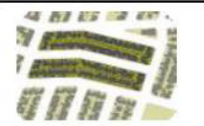 & (1) =1 & end & 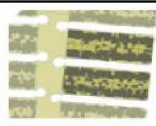 \\
\hline SVF & 0.16 & 0.30 & 0.60 & 0.72 & 0.57 & 0.45 \\
\hline Especie forestal & Tipuana tipu & Morus alba & Ulmus umbraculifera & Melia azedarach & Morus alba & Morus alba \\
\hline Largo (m) & 116 & 163 & 226 & 245 & 145 & 145 \\
\hline Ancho (m) & 20 & 16 & 20 & 16 & 20 & 12 \\
\hline Alto (m) & 3 & 3 & 3 & 3 & 3 & 3 \\
\hline $\mathrm{H} / \mathrm{W}$ & 0.15 & 0.19 & 0.15 & 0.19 & 0.15 & 0.25 \\
\hline$\%$ Construido & 66 & 11 & 32 & 42 & 32 & 27 \\
\hline$\%$ Forestado & 34 & 24 & 36 & 24 & 42 & 18 \\
\hline$\%$ Terreno natural & 0 & 65 & 32 & 34 & 26 & 59 \\
\hline$\%$ Calles & 35 & 44 & 40 & 45 & 37 & 33 \\
\hline$\%$ Manzana & 65 & 56 & 60 & 55 & 63 & 67 \\
\hline Altitud & 727 & 810 & 795 & 850 & 744 & 880 \\
\hline Pendiente & 1.5 & 3.8 & 1.9 & 3.5 & 1 & 4.5 \\
\hline
\end{tabular}

Figura 5. Caracterización e indicadores urbanos de los casos de estudio. Fuente: Elaboración de las autoras.

\section{CASOS DE ESTUDIO EN LLANURA Y PIEDEMONTE}

Para desarrollar el trabajo se seleccionaron y caracterizaron seis CVU insertos en tres formas de tramas: racional, orgánica y Cul-de-Sac; existentes en cada escenario geomorfológico -llanura y piedemonte-. La Figura 4 muestra su ubicación dentro del territorio del AMM. Se define por "forma de trama Cul-de-Sac" a los conjuntos urbanos con calles que poseen un solo punto de entrada y salida, y que finalizan con un ensanche de forma circular que permite girar con el vehículo.

La caracterización de cada caso se efectuó mediante un grupo de indicadores morfológicos (largo y ancho de calle, H/W), forestales (SVF, especie forestal), geomorfológicos (altitud y pendiente) y de cobertura de suelo (\% calles, \% construido, $\%$ manzanas, \% terreno natural y \% forestado). Es importante destacar que cada CVU seleccionado, dentro del universo existente en el AMM, mantiene similares ciertas características. Ahora bien, existen diferencias en algunos de los indicadores urbanos, las cuales se presentan en la sección de resultados el análisis, de manera tal que se pueden contrastar las diversas formas existentes en ambos escenarios geomorfológicos. Los CVU analizados dentro de cada trama poseen una orientación E-O, orientación que, por cierto, es la más perjudicial para las condiciones de habitabilidad de los espacios exteriores debido a su mayor exposición al recorrido solar y a que, además, en condiciones de piedemonte, contribuye a la aceleración de los cauces aluvionales. La Figura 5 expone los valores de los indicadores urbanos de los casos de estudio seleccionados.
La clasificación y cuantificación de la cobertura de suelo de cada caso de estudio se llevó a cabo a partir del procesamiento de imágenes satelitales. Se utilizaron, en concreto, imágenes multi-espectrales de tipo ASTER que son capturadas por el satélite TERRA. A su vez, ASTER posee tres sub-sistemas de sensores: VNIR, SWIR y TIR. El sub-sistema VNIR captura imágenes en tres bandas del rango de luz, en un rango espacial de $15 \mathrm{~m}$. Siendo esta resolución la más óptima en términos de visualización y análisis, ya que permite distinguir en la imagen los objetos con mayor precisión por el tamaño del píxel, medido en metros sobre el terreno (píxel= $15 \times$ $15 \mathrm{~m})$. El procedimiento de clasificación de estas imágenes, con un área de recorte de $560 \times 560 \mathrm{~m}^{2}$, se realizó con el freeware MultiSpec@. Esta herramienta permite procesar y analizar imágenes de tipo hiper-espectral y multi-espectral, e incluye seis algoritmos de clasificación que hacen posible diferenciar el porcentaje existente de píxeles de cada clase con características espectrales similares. Para esta investigación, se optó por el análisis por componentes principales (módulo de análisis discriminante lineal de Fisher). La clasificación de cada imagen se realizó seleccionando por tipo de clase un campo de píxeles: cada clase coincide con las coberturas de suelo anteriormente descriptas.

El indicador SVF fue calculado mediante imágenes hemisféricas capturadas con una cámara digital Nikon ${ }^{\circledR}$ CoolPix equipada con un lente ojo de pez; procesadas con el software Píxel de Cielo. Este software obtiene el valor de SVF en condiciones de cielo despejado, con forestación urbana intensa y en ciudades con alta reflectividad, típica de regiones áridas como es el caso de este estudio. La relación H/W y, ancho y longitud de la calle, fue determinada a partir del relevamiento in situ en cada CVU. 


\begin{tabular}{|c|c|c|c|c|c|c|c|}
\hline Sensor & \multicolumn{6}{|c|}{ Estación Hobo Weather Station ${ }^{\circledR}$} & Fluke 66 \\
\hline Variable & Temperatura & $\begin{array}{c}\text { Humedad } \\
\text { relativa }\end{array}$ & $\begin{array}{l}\text { Velocidad } \\
\text { del viento }\end{array}$ & $\begin{array}{l}\text { Dirección } \\
\text { del viento }\end{array}$ & $\begin{array}{c}\text { Radiación } \\
\text { solar }\end{array}$ & $\begin{array}{c}\text { Presión } \\
\text { atmosférica }\end{array}$ & $\begin{array}{l}\text { Temperatura } \\
\text { superficial }\end{array}$ \\
\hline $\begin{array}{l}\text { Rango de } \\
\text { medición }\end{array}$ & -40 a $75^{\circ} \mathrm{C}$ & 0 a $100 \%$ & 0 a $45 \mathrm{~m} / \mathrm{s}$ & 0 a $355^{\circ}$ & 0 a $1280 \mathrm{~W} / \mathrm{m}^{2}$ & 660 a 1070 mbar & -32 a $600^{\circ} \mathrm{C}$ \\
\hline $\begin{array}{l}\text { Rango de } \\
\text { operación }\end{array}$ & \multicolumn{6}{|c|}{$-40^{\circ} \mathrm{C}$ a $75^{\circ} \mathrm{C}$} & \\
\hline Precisión & $0.2^{\circ} \mathrm{C}$, a $0^{\circ} \mathrm{C}-50^{\circ} \mathrm{C}$ & $\begin{array}{c} \pm 2,5 \%, \text { desde } 10 \text { a } \\
90 \% \mathrm{HR}\end{array}$ & $\pm 1.1 \mathrm{~m} / \mathrm{s} 64 \%$ & $\pm 5^{\circ}$ & $\begin{array}{c} \pm 10.0 \mathrm{~W} / \mathrm{m}^{2} \text { ó } \\
\pm 5 \%\end{array}$ & $\begin{array}{c} \pm 3.0 \text { mbar, sobre el } \\
\text { rango de máxima } \\
\text { presión a } 25^{\circ} \mathrm{C} \text {. }\end{array}$ & $\begin{array}{c} \pm 1 \% \text { de la lectura ó } \\
\pm 1^{\circ} \mathrm{C}\end{array}$ \\
\hline Resolución & 0.02 a $25^{\circ} \mathrm{C}$ & $0.1 \% \mathrm{HR}$ a $25^{\circ} \mathrm{C}$ & $0.38 \mathrm{~m} / \mathrm{s}$ & $1.4^{\circ}$ & $1.25 \mathrm{~W} / \mathrm{m}^{2}$ & $0.1 \mathrm{mbar}$ & \\
\hline
\end{tabular}

\section{MONITOREO MICROCLIMÁTICO}

Con el fin de conocer el comportamiento microclimático de los seis casos de estudio, se monitoreó mediante campañas de mediciones con sensores fijos (Hobo® H08-003-02) ubicados en cada CVU y dos estaciones meteorológicas móviles (Hobo Weather Station $®)$. El periodo de medición fue entre el 22 de enero al 24 de febrero de 2016. En las mediciones fijas, los sensores se instalaron a $2 \mathrm{~m}$ de altura desde el nivel de la calle dentro de una caja perforada de PVC blanco con el objeto de evitar la irradiación y asegurar una adecuada circulación de aire. En las mediciones móviles se utilizó una estación en cada CVU, la cual fue instalada sobre un soporte móvil capaz de desplazarla por 4 puntos de monitoreo para tomar los valores a lo largo de una hora en cada CVU. El periodo de medición con estaciones móviles fue desde las 10:00 h hasta las 20:00 h, por una situación de logística y con el propósito de preservar la seguridad del equipamiento. Simultáneamente, se registraron los valores de temperatura superficial de paredes, veredas y calle con un termómetro infrarrojo (Fluke 66). Los datos de la campaña fueron adquiridos cada 15 minutos. La Figura 6 describe el equipamiento de medición utilizado.

\section{CONFORT TÉRMICO EXTERIOR}

A nivel mundial existen varios modelos que sirven para conocer y calcular el grado de confort térmico exterior: PET (Matzarakis, Helmut y Moses, 1999), PMV (Fanger, 1970), COMFA (Brown y Gillespie, 1995), entre otros. En este trabajo las condiciones de confort de cada CVU fueron determinadas mediante el modelo COMFA.

Para el caso de estudio del AMM, y su condicionante de ser una "ciudad oasis", el modelo COMFA resulta ser el indicador más adecuado, ya que posibilita una mayor diferenciación de las características urbanas y forestales, en adición a las variables climáticas consideradas por los demás índices (Ruiz y Correa, 2014). El modelo COMFA resuelve una ecuación de balance de energía $\mathrm{S}$, siendo el rango de confort térmico $S$ entre -50 a $50 \mathrm{~W} / \mathrm{m}^{2}$. Si el balance presenta un gran valor positivo, la persona recibe más energía que la que pierde, por lo que podría haber sobrecalentamiento y estaría inconfortable a causa del calor. Por otra parte, si el balance es negativo, la persona podría sentir frío. A continuación, se presenta la fórmula del balance de energía S:

$\mathrm{S}=\mathrm{M}+\mathrm{R}_{\mathrm{abs}}-$ Conv - Evap $-T R_{\text {emitida }}$

Donde: M energía metabólica producida por el organismo; Rabs radiación solar y terrestre absorbida; Conv calor sensible perdido o ganado por convección; Evap pérdida evaporativa de calor; TRemitida radiación terrestre emitida. Los valores de los parámetros de cada uno de los cinco términos de la fórmula $\mathrm{S}$ fueron obtenidos del monitoreo microclimático; $y$, asimismo, se definieron valores para las variables forestales, materiales del espacio construido y de condiciones de exposición de los usuarios, que intervienen en el método COMFA. De esa forma, se determinó lo siguiente:

- Permeabilidad de los forestales: Tipuana Tipu 0.12, Melia Azedarach 0.28, Morus Alba 0.31, Ulmus Umbraculifera 0.15 (magnitud adimensional)

- Albedo promedio superficies verticales: 0.40 (magnitud adimensional)

- Albedo promedio superficies horizontales: 0.10 (magnitud adimensional)

- Aislamiento vestimenta: $75 \mathrm{clo}$

- Permeabilidad vestimenta: 150 (magnitud adimensional)

- Tasa metabólica: $120 \mathrm{~W} / \mathrm{m}^{2}$

\section{CORRELACIÓN ESTADÍSTICA.}

A partir de la caracterización de los seis casos de estudio, mediante los once indicadores urbanos anteriormente descritos (Figura 5), se efectuó un análisis estadístico. El método seleccionado para tal labor fue el de correlación con el coeficiente de Pearson. Este índice es una medida 


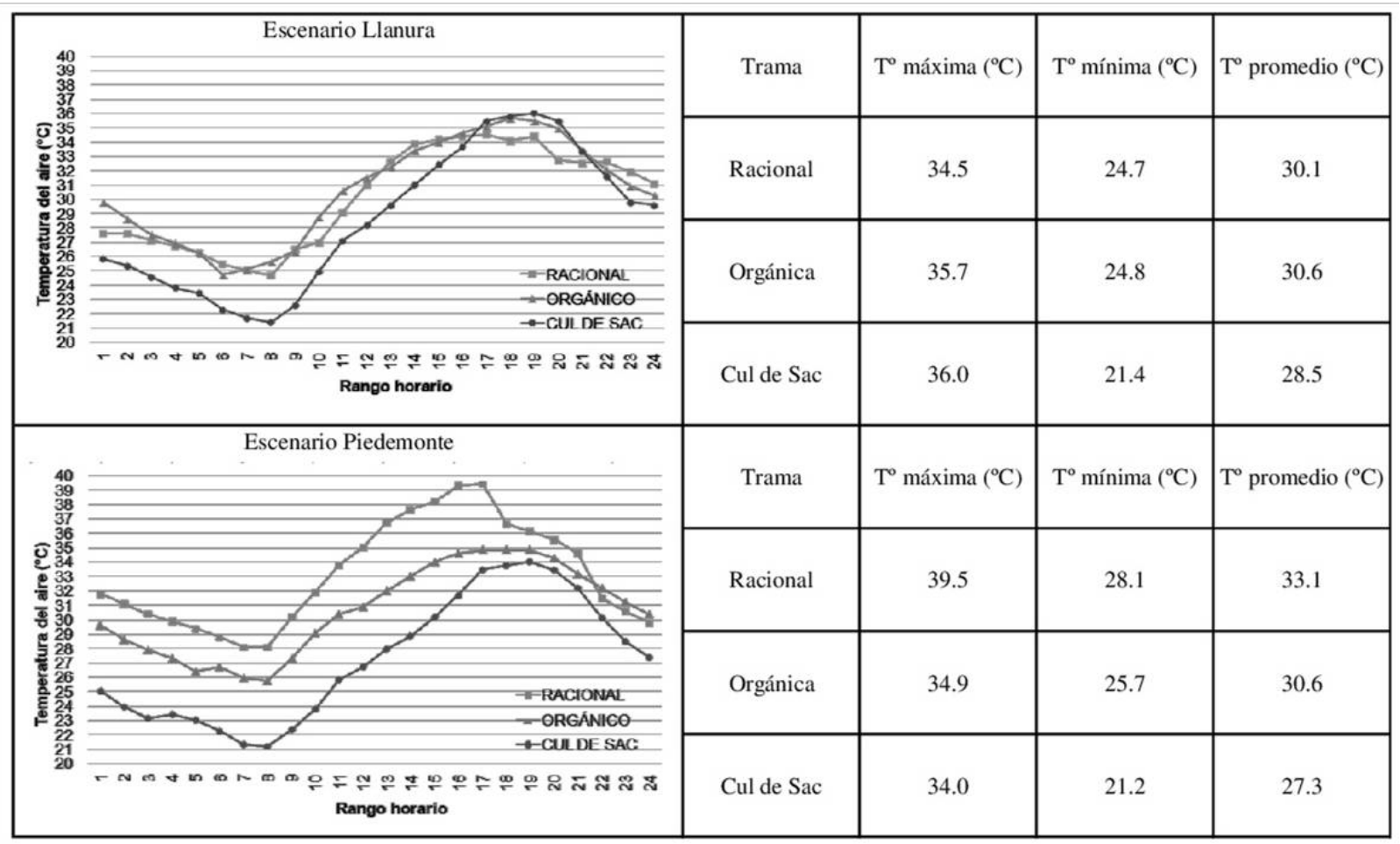

Figura 7. Comportamiento térmico, contraste entre escenarios de llanura y piedemonte. Fuente: Elaboración de las autoras.

lineal e independiente de la escala que permite medir el grado de relación entre dos variables cuantitativas; donde su magnitud indica el grado de asociación entre las variables y los valores absolutos oscilan entre -1 y +1 . En este estudio se considera una relación de magnitud de +/-0.5, según corresponda, como significativa. La matriz de correlación se calculó mediante el uso del software InfoStat (Di Rienzo et al., 2011).

A través del análisis recién mencionado fue posible reconocer cómo ciertos indicadores urbanos muestran relación entre sí (mayor a 0.5) para alcanzar mejoras en los aspectos térmicos y de confort del espacio exterior, a efectos de generar recomendaciones de diseño urbano para los planificadores.

\section{RESULTADOS}

\section{COMPORTAMIENTO TÉRMICO}

Como resultado del monitoreo microclimático, se determinó que la respuesta térmica varía al contrastar los escenarios geomorfológicos de inserción, a pesar de replicarse la forma de trama urbana en ambos sectores.

La Figura 7 exhibe las curvas de comportamiento térmico de los seis CVU monitoreados. Se seleccionó el día 23 de enero de 2016 ya que es representativo de las condiciones climáticas del AMM (día claro, elevada heliofanía, baja velocidad de vientos y baja humedad relativa).

Al analizar y contrastar el comportamiento de los casos, se visualiza que:
- El CVU inserto en la trama racional es más caluroso en el piedemonte que en la llanura; registrándose diferencias entre las temperaturas máximas $\left(\triangle 4.9^{\circ} \mathrm{C}\right)$, mínimas $\left(\triangle 3.4^{\circ} \mathrm{C}\right)$ y promedios $\left(\triangle 2.9^{\circ} \mathrm{C}\right)$.

- El CVU inserto en la trama orgánica tiene la temperatura máxima más elevada en la llanura, con una diferencia de $0.8^{\circ} \mathrm{C}$ respecto al piedemonte, sin embargo, la temperatura mínima del CVU inserto en la trama orgánica de llanura es $1.0^{\circ} \mathrm{C}$ más fresco respecto al CVU en el piedemonte. Las temperaturas promedio, en ambos escenarios, son muy similares $(\Delta$ $\left.0.1^{\circ} \mathrm{C}\right)$.

- El CVU inserto en la trama Cul-de-Sac es más caluroso en la llanura que en el piedemonte, dándose diferencias entre sus temperaturas máximas $\left(\triangle 2.0^{\circ} \mathrm{C}\right)$, mínimas $\left(\triangle 0.2^{\circ} \mathrm{C}\right)$ y promedios $\left(\triangle 1.3^{\circ} \mathrm{C}\right)$.

Los CVU insertos en la trama Cul-de-Sac muestran mayor refrescamiento nocturno en ambos escenarios geomorfológicos. Asimismo, se observa una mayor diferenciación en el comportamiento térmico de los CVU localizados en el piedemonte, lo que parecería indicar que el impacto de la forma de la trama sobre el desempeño térmico del espacio es más importante en esta geomorfología.

\section{CONFORT TÉRMICO EXTERIOR.}

La Figura 8 ilustra los porcentajes acumulados de cada rango de sensación del balance COMFA, siendo el color verde el rango donde las personas se sienten en confort térmico. De este resultado se desprende que: 


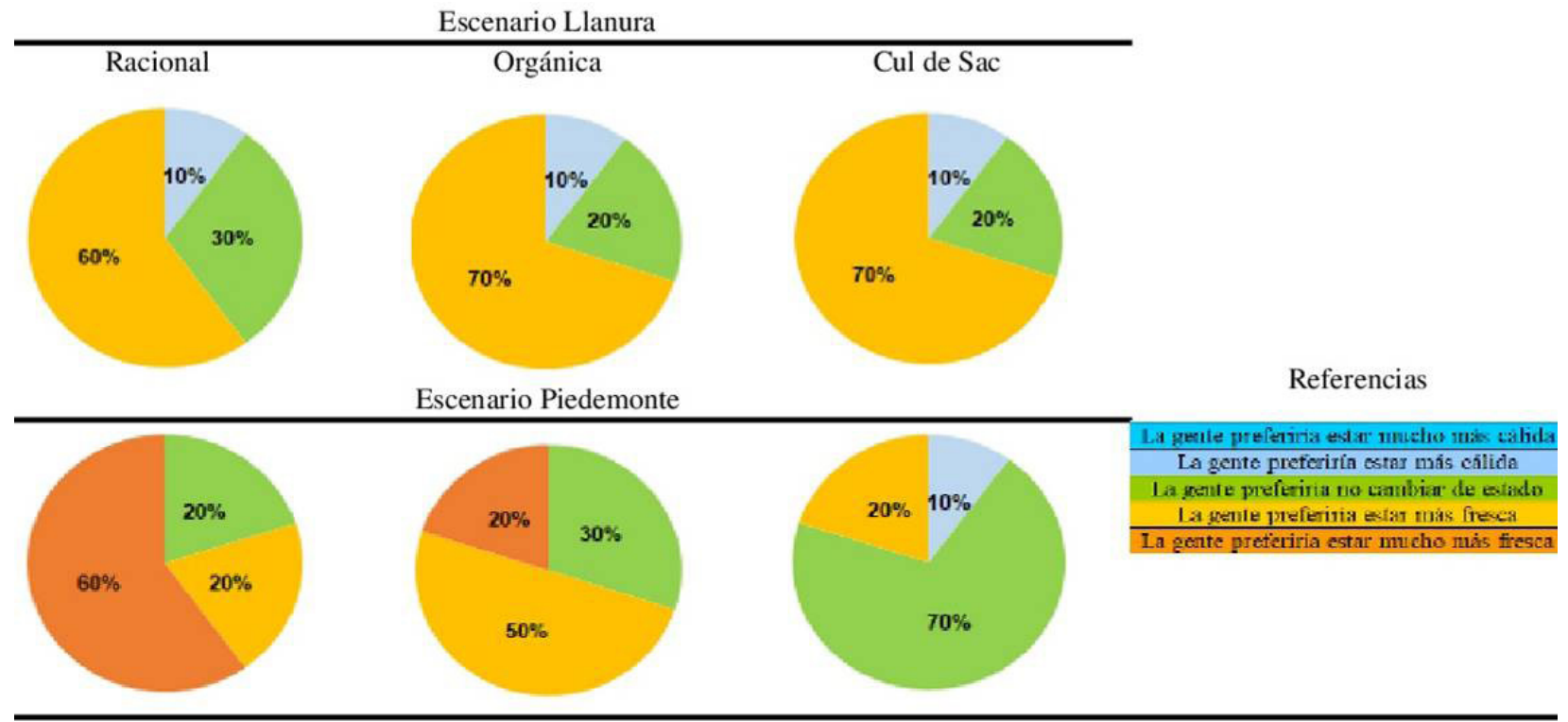

Figura 8. Distribución de la frecuencia (\%) en las condiciones de confort térmico en cada caso evaluado. Fuente: Elaboración de las autoras

\begin{tabular}{|l|c|c|c|c|c|c|}
\hline & \multicolumn{2}{|c|}{ Racional } & \multicolumn{2}{c|}{ Orgánica } & \multicolumn{2}{c|}{ Cul de Sac } \\
\hline Escenario Llanura & $\mathbf{+}$ & $\mathbf{-}$ & $\mathbf{-}$ & $\mathbf{-}$ & $\mathbf{+}$ \\
\hline Escenario Piedemonte & $\mathbf{-}$ & $\mathbf{-}$ & $\mathbf{+}$ & $\mathbf{+}$ & $\mathbf{+}$ & $\mathbf{+}$ \\
\hline Respuesta & $\begin{array}{c}\text { Comportamiento } \\
\text { térmico }\end{array}$ & Confort térmico & $\begin{array}{c}\text { Comportamiento } \\
\text { térmico }\end{array}$ & Confort térmico & $\begin{array}{c}\text { Comportamiento } \\
\text { térmico }\end{array}$ & Confort térmico \\
\hline
\end{tabular}

Figura 9. Contraste de cada trama analizada según su entorno de inserción. Fuente: Elaboración de las autoras.

- En el sector de llanura, los CVU pertenecientes a la trama racional alcanzan, durante un $30 \%$ del tiempo evaluado, condiciones de confort; mientras que en el sector de piedemonte ello representa un $20 \%$.

- En el sector de piedemonte, los CVU pertenecientes a la trama orgánica logran condiciones de confort, durante un $30 \%$ del tiempo evaluado; mientras que en el sector de llanura este porcentaje corresponde a un $20 \%$.

- En el sector de piedemonte, los CVU pertenecientes a la trama Cul-de-Sac alcanzan condiciones de confort, durante un $70 \%$ del tiempo evaluado; mientras que en el sector de llanura esta situación representa sólo un $20 \%$.

Estas cifras evidencian que:

- Los CVU insertos en la trama de tipo racional, de uso extensivo en las urbanizaciones de ambos escenarios geomorfológicos, presentan mayor disconfort térmico en el piedemonte. Es importante subrayar que este tipo de trama se contradice con las recomendaciones internacionales para el diseño urbano de las áreas de piedemonte que aconsejan respetar la topografía del terreno natural utilizando tramas orgánicas, con una distribución tipo "cluster" y el esquema de Cul-de-Sac en sus CVU (Castillo, Correa y Cantón, 2017).

- Los CVU insertos en la trama de tipo Cul-de-Sac u orgánica, que se adaptan a las condiciones naturales del terreno en el sector de piedemonte, muestran mejores condiciones de habitabilidad térmica exterior.

\section{COMPORTAMIENTO TÉRMICO Y CONFORT EXTERIOR}

La Figura 9 resume y contrasta la respuesta térmica y las condiciones de confort exterior en cada CVU inserto en diferentes tramas y escenarios geomorfológicos. El signo + + indica el desempeño óptimo, que se define como el que registra temperaturas máximas y mínimas más bajas y, mayor frecuencia de confort. El signo - . señala el comportamiento más desfavorable, es decir, el que presenta temperaturas máximas y mínimas más altas $y$, mayor frecuencia de disconfort. Los signos $+y$ - expresan condiciones intermedias.

La Figura 9 muestra que la respuesta térmica y las 


\begin{tabular}{|l|c|c|c|c|}
\hline & $\mathrm{T}^{\circ}$ máxima & $\mathrm{T}^{\circ}$ mínima & $\mathrm{T}^{\circ}$ promedio & COMFA \\
\hline Largo & 0.0 & 0.4 & 0.3 & -0.3 \\
\hline Ancho & 0.1 & 0.1 & 0.2 & $-0.8^{*}$ \\
\hline $\mathrm{H} / \mathrm{W}$ & -0.3 & -0.3 & $-0.5^{*}$ & $0.9^{*}$ \\
\hline SVF & -0.2 & -0.2 & -0.2 & -0.1 \\
\hline Altitud & -0.1 & 0.0 & -0.1 & $0.6^{*}$ \\
\hline Pendiente & 0.1 & 0.2 & 0.1 & $0.6^{*}$ \\
\hline$\%$ calles & $0.6^{*}$ & $0.8^{*}$ & $0.8^{*}$ & $0.6^{*}$ \\
\hline$\%$ construído & $-0.7^{*}$ & -0.2 & -0.2 & 0.0 \\
\hline$\%$ manzanas & $-0.6^{*}$ & $-0.8^{*}$ & $-0.8^{*}$ & $-0.6^{*}$ \\
\hline$\%$ terreno natural & $0.5^{*}$ & 0.2 & 0.2 & 0.3 \\
\hline$\%$ forestado & 0.0 & -0.2 & 0.0 & $-0.7^{*}$ \\
\hline
\end{tabular}

Figura 10. Coeficientes de correlación de Pearson, $* R^{2} \geq 0.5$. Fuente: Elaboración de las autoras.

condiciones de confort de un CVU inserto en una misma trama urbana tienen un comportamiento diferente de acuerdo a la geomorfología en la cual se inserta. Por ejemplo, el desempeño del CVU dispuesto en una trama racional muestra un comportamiento positivo en escenarios de llanura y muy desfavorable en escenarios de piedemonte. Estos resultados ponen en relieve la necesidad de estudiar el efecto combinado de las características del perfil urbano, la trama en la que se incorpora y la geomorfología de localización.

\section{RECOMENDACIONES DE DISEÑO URBANO.}

Los análisis previos han permitido determinar comportamientos térmicos y condiciones de confort de diferentes CVU insertos en distintos tipos de trama y contextos geomorfológicos. Sin embargo, los CVU analizados evidencian ligeras diferencias en los indicadores utilizados para su caracterización que dificultan un análisis univariado para establecer relaciones entre variables de diseño y respuesta térmica. En este sentido, el análisis estadístico persigue identificar el impacto de los indicadores en la respuesta térmica y las condiciones de confort de los distintos casos, a fin de definir pautas o recomendaciones de diseño tendientes a disminuir las temperaturas urbanas y mejorar las condiciones de confort.

La Figura 10 grafica los coeficientes de correlación de Pearson entre las variables respuesta (temperaturas del aire y confort) y las variables independientes (indicadores urbanos). El análisis de los resultados indica que existen correlaciones de significancia estadística con ocho indicadores -identificados con un asterisco-.
El análisis de las correlaciones revela, respecto a las temperaturas del aire, que las temperaturas máximas correlacionan positivamente con los siguientes indicadores: superficie de calle y terreno natural; y negativamente, con la superficie construida y la superficie de manzana. Es decir, las temperaturas máximas se incrementan cuando crece la superficie de calle y de terreno natural, y disminuye la superficie construida y la de la manzana.

Las temperaturas mínimas muestran una correlación positiva con la superficie de calle y negativa con la superficie de manzana. Esto significa que las temperaturas mínimas aumentan cuando crece la superficie de calle y disminuye la superficie de manzana.

Respecto a las temperaturas promedio, estas correlacionan positivamente con la superficie de calle y negativamente con la superficie de manzana y el H/W.

Finalmente, los valores de COMFA correlacionan positivamente con la altitud, pendiente, superficie de calle y H/W. Y lo hacen negativamente con el ancho de calle, superficie de manzana y superficie forestada. Debe tenerse en cuenta que valores de COMFA más altos refieren a mayor disconfort térmico, o sea, que las condiciones de confort térmico mejoran cuando disminuye la altitud, pendiente, superficie de calle y H/W; y se empobrecen cuando disminuye el ancho de calle, superficie de manzana y superficie forestada.

De lo aquí expuesto, se desprende que el confort se encuentra condicionado, entre otras variables, por la geomorfología. Como se dijo previamente, el confort 
térmico disminuye cuando aumenta la altitud y la pendiente, características dominantes del piedemonte. Por otra parte, disminuye cuando aumenta la superficie de calle y el H/W. De la combinación del conjunto de variables se infiere que, a mayores exigencias del relieve, asociadas a la condición de piedemonte, es necesario tender a la compacidad del modelo urbano para mejorar las condiciones de confort.

\section{CONSIDERACIONES FINALES}

Este trabajo ha permitido determinar el comportamiento térmico y las condiciones de confort de CVU insertos en diferentes tipos de trama y geomorfologías, mediante el monitoreo de las condiciones microclimáticas. Asimismo, ha sido posible analizar la influencia de los indicadores que caracterizan dichos espacios con el desempeño térmico y las condiciones de confort de los mismos a través del análisis estadístico de los datos.

Los resultados obtenidos demuestran que la respuesta térmica y el grado de confort de los CVU implantados en una misma trama urbana tienen diferentes comportamientos de acuerdo a la geomorfología de implantación. En consecuencia, el estudio avala la necesidad de abordar el efecto combinado de las características del perfil urbano, la trama en la que se inserta y la geomorfología de localización. Esto representa un avance en la generación de conocimiento de acuerdo al estado del arte.

En Ilanura, el CVU de trama racional registra los mejores desempeños, mientras que en piedemonte el mejor desempeño, tanto térmico como de confort exterior, está asociado al CVU de trama Cul-de-Sac, y en menor grado a los CVU de trama orgánica. Por lo tanto, en el caso de relieves signados por mayor altitud y pendiente, la compacidad de la forma genera beneficios asociados a la disminución de las temperaturas máximas, mínimas y promedio, y a la mejora de las condiciones de confort. Esto se traduce en la necesidad de desarrollar tramas con menores superficies de calles, mayores superficies de manzanas y forestación, y bajas densidades constructivas.

En definitiva, el confort se encuentra condicionado, entre otras variables, por la geomorfología dado que los valores de COMFA correlacionan positivamente con la altitud y la pendiente. Es decir, en piedemonte, a igualdad de valores de otros indicadores en llanura, el confort térmico disminuye. En este sentido, la forma de urbanizar el territorio debe considerar las características propias de cada sitio, sobre todo cuando se evidencia que los trazados de la urbanización del piedemonte han continuado las tramas desarrolladas en la llanura, tramas en su mayoría racionales, las cuales, según los datos del presente trabajo, muestran precisamente el peor desempeño en tal sector, tanto en su comportamiento térmico como en el grado de habitabilidad exterior.
Finalmente, hay que recordar que la necesidad de aumentar las tierras urbanizables, debido al aumento de población, impulsa el proceso de expansión urbana. De acuerdo a la tendencia de desarrollo del AMM y el marco regulatorio de los trazados urbanos, se hace patente que el mismo no ha sido planificado ni cuenta con normativas que contemplen la incidencia de la geomorfología en la eficiencia del funcionamiento térmico de las urbanizaciones. Por consiguiente, las conclusiones de este trabajo aspiran a contribuir al ámbito de la planificación a escala regional, como también a reflejar la importancia de abordar la problemática del desarrollo urbano desde un enfoque que considere y valorice la definición de la forma urbana en función de las particularidades del territorio a intervenir.

\section{AGRADECIMIENTOS}

El presente trabajo se llevó a cabo gracias a los recursos financiados por el Consejo Nacional de Investigaciones Científicas y Técnicas (CONICET) [PIP 2011-00640] y por la Agencia Nacional de Promoción Científica y Tecnológica (ANPCyT) [PICT 2011-0611].

\section{REFERENCIAS BIBLIOGRÁFICAS}

ABRAHAM, Elena. Proyecto: Planificación y Ordenamiento Ambiental del Piedemonte al Oeste de la Ciudad de Mendoza. IADIZA, 1990.

ABRAHAM, Elena. Mapa Geomorfológico de la Provincia de Mendoza en escala 1:500.000. En: Atlas básico de recursos de la región andina argentina. Informe Final. Enviado a Universidad de Granada, Junta de Gobierno Andalucía, España, 1996.

AKBARI, Hashem; CARTALIS, Constantinos; KOLOKOTSA, Denia; MUSCIO, Alberto; PISELLO, Anna Laura; ROSSI, Federico; SANTAMOURIS, Matheos; SYNNEFA, Afroditi; WONG, Nyuk y ZINZI, Michele. Local climate change and urban heat island mitigation techniques. The estate of the art, Journal of Civil Engineering and Managment, 2016, vol. 22, pp. 1-16.

ALBĂ, Claudia; MITITELU-IONUŞ, Oana y BOENGIU, Sandu. "Geomorphological facilities and constraints in urban expansion of Craiova city". En: Conference: Proceedings of the Romanian Geomorphology Symposium, 2017, edición 33, pp. 11-14

ALCHAPAR, Noelia y CORREA, Érica. Comparison the performance of different facade materials for reducing building cooling needs. En: PACHECO-TORGAL, Fernando; LABRINCHA, Joao; CABEZA, Luisa y GRANQVIST, Claes (eds.). Eco-efficient materials for mitigating building cooling needs. Cambridge: Woodhead, 2015, pp. 155-194.

ANGEL, Shlomo; PARENT, Jason; CIVCO, Daniel; BLEI, Alexander y POTERE, David. The dimensions of global urban expansion: Estimates and projections for all countries, 20002050, Progress in Planning, 2011, 75 n² 2, pp. 53-107. 
BARAKAT, Asmaa, AYAD, Hany y EL-SAYED, Zeyad. Urban design in favor of human thermal comfort for hot arid climate using advanced simulation methods, Alexandria Engineering Journal, 2017, vol. 56, n², pp. 533-543.

BROWN, Robert y GILLESPIE, Terry. Microclimate Landscape Design. Somerset, New Jersey, U.S.A: John Wiley \& Sons Inc., 1995

CASTALDO, Verónica; PISELLO, Anna; PIGLIAUTILE, Ilaria; PISELLI, Cristina y COTANA, Franco. Microclimate and air quality investigation in historic hilly urban areas: Experimental and numerical investigation in central Italy, Sustainable Cities and Society, 2017 vol. 33, pp. 27-44.

CASTILLO, Ana Laura, CORREA, Érica y CANTÓN, María Alicia. Regulación urbanística de áreas de piedemonte en ciudades latinoamericanas. Análisis crítico de la normativa internacional y el marco regulatorio de la ciudad de Mendoza, Argentina, ACE: Architecture, City and Environment. Arquitectura, Ciudad y Entorno, 2017, vol. 12, pp. 39-70.

CASTRILLÓN, Alberto y CARDONA, Sandra. El urbanismo y la planeación moderna. Glocalidades en la formación de la modernidad urbana de Medellín, Revista Historia y Sociedad, 2014, vol. 26, pp. 17-51

CAVALLARO, Sandra; NICOSIA BURGOS, Federico y FONTANETO, Patricio Julián. La cartografía ambiental como base para los estudios de planificación ecológica del territorio, Asociación Geológica Argentina, 2010, vol. 66, pp. 475-483.

CORREA, Érica; DE ROSA, Carlos y LESINO, Graciela. Urban heat island effect on heating and cooling degree day's distribution in Mendoza's metropolitan area. Environmental costs. En: SOCIEDADE PORTUGUESA DE ENERGIA SOLAR (SPES) (ed.). Proceedings of the EUROSUN 2008. NY, USA: Curran associates, Inc. Red Hook, pp. 951-958.

CORREA, Érica; RUIZ, María Angélica y CANTÓN, María Alicia. Morfología forestal y confort térmico en "ciudades oasis" de zonas áridas, Ambiente Construido, 2010, vol.10, n² 4, pp.119137

DELGADO, Gian Carlo; CAMPOS CHÁVEZ, Cristina y RENTERÍA JUÁREZ, Patricia. Cambio climático y el metabolismo urbano de las Megaurbes Latinoamericanas, Hábitat Sustentable, 2012, vol. 2, $n^{\circ} 1$, pp. 2-25.

DI RIENZO, Julio; CASANOVES, Fernando; BALZARINI, Mónica; GONZÁLEZ, Laura; TABLADA, Margot; ROBLEDO, Walter. InfoStat v. 2011. Grupo InfoStat, FCA, Universidad Nacional de Córdoba, Argentina, 2011.

DOBBS, Cynnamon; HERNÁNDEZ MORENO, Ángela; REYES PAECKE, Sonia y MIRANDA, Marcelo. Exploring temporal dynamics of urban ecosystem services in Latin America. The case of Bogotá (Colombia) and Santiago (Chile), Ecological Indicators, 2018, vol. 85, pp. 1068-1080.

EMMANUEL, Rohinton y FERNANDO, H. Urban heat islands in humid and arid climates: role of urban form and thermal properties in Colombo, Sri Lanka and Phoenix, USA, Climate Research, 2007, vol. 34, n³, pp. 241-251.
ENCINAS, Felipe; MARMOLEJO, Carlos y AGUIRRE, Carlos. El impacto de los proyectos inmobiliarios y sus atributos de sustentabilidad sobre el valor del suelo: ¿causa o consecuencia? Dos estudios de casos para Santiago de Chile, Hábitat Sustentable, 2016, vol. 6, n² 2, pp.70-79.

FANGER, Ole. Thermal Comfort: Analysis and applications in environmental engineering. Copenhagen: Danish Technical Press, 1970, pp. 244.

GIVONI, Baruch. Urban design in different climates. World meteorological organization, 1989.

GOLDEN, Jay. The Built Environment Induced Urban Heat Island Effect in Rapidly Urbanizing Arid Regions. A Sustainable Urban Engineering Complexity, Environmental Sciences, 2004 vol. 1, n 4, pp. 321-349.

GÓMEZ, Nersa; HIGUERAS, Ester y FERRER, Mercedes. Análisis del Confort Térmico Social para el Control sostenible del Microespacio Urbano entre edificaciones, Revista Portafolio, 2016, vol. 2, n³4, pp. 59-70.

GUZMÁN, Francisco y OCHOA DE LA TORRE, José. Confort térmico en los espacios públicos urbanos. Clima cálido y frío semi-seco, Hábitat Sustentable, 2014, vol. 4, n² 2, pp. 52-63.

KO, Yekang y RADKE, John. The Effect of Urban Form and Residential Cooling Energy Use in Sacramento, California, Environment and Planning B: Urban Analytics and City Science, 2014, vol. 41, n 4, pp. 573- 593.

LEVERMORE, Geoff; PARKINSON, John; LEE, Kwanho; LAYCOCK, Patrick y LINDLEY, Sarah. The increasing trend of the urban heat island intensity, Urban Climate, 2018, vol. 24 pp. 360-368.

LIBERTUN DE DUREN, Nora. Urbanización rápida y desarrollo: cumbre de América Latina y China. En: Cumbre de América Latina y China de Políticas y Conocimiento: Impactos de la urbanización rápida y la prosperidad nacional (Lima, 15 y 16 de julio de 2014) [en línea]. Banco Inter-Americano del Desarrollo, 2014, pp. 9-11. [Consultado 12 octubre 2018]. Disponible en: https://publications.iadb.org/handle/11319/6732

LIN, Tzu-Ping; MATZARAKIS, Andreas y HWAND, Ruey-Lung. Shading effect on long-term outdoor thermal comfort, Building and Environment, 2011, vol. 45, pp. 213-211.

MATZARAKIS, Andreas; HELMUT, Mayer y MOSES, Iziomon. Applications of a universal thermal index: physiological equivalent temperature, Int J Biometeorol, 1999, vol. 43, pp.76-84

MAWROMATIS, Constantino. Tensiones y convergencia: el diseño urbano contemporáneo como alternativa a la ciudad dispersa y difusa, Revista INVI, 2013, vol. 28, n 79, pp. 125 163.

MIDDEL, Ariane; HÄB, Kathrin; BRAZEL, Anthony; MARTIN, Chris y GUHATHAKURTA, Subhrajit. Impact of urban form and design on mid-afternoon microclimate in Phoenix Local Climate Zones, Landscape and Urban Planning, 2014, vol. 122, pp. 16-28. 
MIDDEL, Ariane; CHHETRI, Nalini y QUAY, Ray. Urban forestry and cool roofs: Assessment of heat mitigation strategies in Phoenix residential neighborhoods, Urban Forestry \& Urban Greening, 2015, vol. 14, pp. 178-186.

MINVU - Ministerio de Vivienda y Urbanismo. Manual de elementos urbanos sustentables tomo I: Sustentabilidad en el espacio público y recomendaciones para Chile, versión $n^{\circ} 2$. Santiago: MINVU, 2017.

MOHAMMED, Bakarman y CHANG, Jae. The influence of height/width ratio on urban heat island in hot-arid climates, Procedia Engineering, 2015, vol. 118, pp. 101-108.

Multispec software (v.9). School of Electrical and Computer Engineering Purdue University [en línea]. [Consultado 8 julio 2018]. Disponible en: https://engineering.purdue.edu/ biehl/ MultiSpec/

NIKOLOPOULOU, Marialena y LYKOUDIS, Spyros. Thermal comfort in outdoor urban spaces: analysis across different European countries, Building and environment, 2006, vol. 141, pp.1455-1470.

OKE, Timothy. The energetic basis of the urban heat island, Quarterly Journal of the Royal Meteorological Society, 1982, vol. 455, pp. 1-24.

PEARLMUTTER, David; BITAN, Arieh y BERLINER, Pedro. Microclimatic analysis of "compact" urban canyons in an arid zone, Atmospheric Environment, 1999, vol. 33, pp. 4143-4150.

RUIZ, María Angélica y CORREA, Érica. Suitability of different comfort indices for the prediction of thermal conditions in tree-covered outdoor spaces in arid cities, Theoretical Applied Climatology, 2014, vol. 122, pp. 69-83.

RUIZ, Angélica; SOSA, Belén; CORREA, Érica y CANTÓN, Alicia. Suitable configurations for forested urban canyons to mitigate the UHI in the city of Mendoza, Argentina. Urban Climate, 2015, vol. 14, pp. 197-212.

RUIZ, Angélica; SOSA, Belén; CORREA, Érica y CANTÓN, Alicia. Design tool to improve daytime thermal comfort and nighttime cooling of urban canyons, Urban Planning and Landscape, 2017, vol. 167, pp. 249-256.

SALAS ESPARZA, María Goretti y HERRERA SOSA, Luis Carlos. La vegetación como sistema de control para las islas de calor urbano en Ciudad Juárez, Chihuahua, Hábitat Sustentable, 2017, vol. 7, $\mathrm{n}^{\circ}$ 1, pp. 14-23.

SANUSI, Ruzana; JOHNSTONE, Denise; MAY, Peter y LIVESLEY, Stephen. Street orientation and side of the street greatly influence the microclimatic benefits street trees can provide in summer, Journal of Environmental Quality, 2016, vol. 45, pp. $167-174$

SETAIH, Khalid; HAMZA, Neveen y TOWNSHEND, Tim. Assessment of outdoor thermal comfort in urban microclimate in hot arid areas. En: 13th International Conference of International Building Performance Simulation Association, Chambery, France, 2013.
SHASHUA-BAR, Limor y HOFFMAN, Milo. Geometry and orientation aspects in passive cooling of canyon streets with trees, Energy and Buildings, 2003, vol. 35, n 1, pp. 61-68.

SHASHUA-BAR, Limor; PEARLMUTTER, David y ERELL, Erell. The cooling efficiency of urban landscape strategies in a hot dry climate, Landscape and Urban Planning, 2009, vol. 92, pp. 179-186.

SICLARI BRAVO, Paola. Desafíos urbanos latinoamericanos y caribeños de cara a los objetivos de desarrollo sustentable y a la nueva agenda urbana, Revista INVI, 2017, vol.32, n 89, pp. 199-211.

SOSA, Belén; CORREA, Érica y CANTÓN, Alicia. Urban grid forms as a strategy for reducing heat island effects in arid cities, Sustainable Cities and Society, 2017a, vol. 32, pp. 547-556.

SOSA, María Belén; CORREA, Érica y CANTÓN, María Alicia. Influencia de la morfología urbana sobre la habitabilidad térmica exterior en una ciudad de clima árido, Hábitat Sustentable, 2017b, vol. 7, n 1, pp. 44-53.

STOCCO, Susana; CANTÓN, María Alicia y CORREA, Érica. Design of urban green square in dry areas: Thermal performance and comfort, Urban Forestry \& Urban Greening, 2015, $n^{\circ} 14$, pp. 323-335.

SUN, Shibo; XU, Xiyan; LAO, Zhaoming; LIU, Wei; LI, Zhandong; HIGUERAS, Ester; HE, Li y ZHU, Jianning. Evaluating the impact of urban green space and landscape design parameters on thermal comfort in hot summer by numerical simulation, Building and Environment, 2017, vol. 123, pp. 277-288.

TALEGHANI, Mohammad; KLEEREKOPER, Laura; TENPIERIK, Martin y VAN DEN DOBBELSTEEN, Andy. Outdoor thermal comfort within five different urban forms in the Netherlands, Building and Environment, 2015, n 83, pp. 65-78.

TUMINI, Irina. El microclima urbano en los espacios abiertos. Estudio de casos en Madrid. Tesis doctoral inédita, Universidad Politécnica de Madrid, 2013.

TUMINI, Irina y PÉREZ, Alexis. Aplicación de los sistemas adaptativos para la evaluación del confort térmico en espacios abiertos, en Madrid, Hábitat Sustentable, 2015, vol. 5, pp. 5567.

TUMINI, Irina; HIGUERAS, Ester y BAERESWYL RADA, Sergio. Urban microclimate and thermal comfort modelling: Strategies for urban renovation, International Journal of Sustainable Building Technology and Urban Development, 2016, vol. 1, nº 7, pp. 1-16.

UN-HÁBITAT. The relevance of street patterns and public space in urban, 2013.

UN-HÁBITAT. Informe de los Objetivos de Desarrollo Sostenible 2016.

VICH, Alberto; LOÓPEZ RODRÍGUEZ, Mariela; LAURO; Carolina y VACCARINO, Emilce. Proposals for Flashflood Management in Western Argentina. Case Study: The Metropolitan Area of Greater Mendoza, Urban Studies [en línea], 2014, vol. 2, pp. 37-48. DOI: http://dx.doi.org/10.4236/cus.2014.21004 
VILLEGAS, Ernesto; CIFUENTES, Alejandra; CONTRERAS, Diana y FERNÁNDEZ, Libardo. Ordenamiento territorial como instrumento, para la zonificación ambiental a través de la Estructura Ecológica Principal, como apoyo a la formulación de los POTs y los POMCAS en Colombia, Revista de Tecnología, 2015, vol. 14, n², pp. 49-76.

WANG Jiong y OUYANG, Wanlu. Attenuating the surface Urban Heat Island within the Local Thermal Zones through land surface modification, Journal of Environmental Management, 2016, vol. 187, pp. 239-252. 